\title{
Engel, Alexander: Risikoökonomie. Eine Geschichte des Börsenterminhandels, 542 S., Campus, Frankfurt a.M. u.a. 2021.
}

\section{Ralf Ahrens}

Angenommen: 3. Dezember 2021 / Online publiziert: 24. Januar 2022

(C) Der/die Autor(en) 2022

Terminbörsen sind ein Spezifikum des modernen Kapitalismus. Im Unterschied zum Effektivhandel werden dort Geschäfte abgeschlossen, deren Erfüllung in die Zukunft verschoben wird. Alexander Engels Habilitationsschrift analysiert die lange Geschichte dieses Phänomens als Umgang mit Risiken: Terminhandel war ein Instrument zur Ausschaltung von Preisänderungsrisiken in der Zukunft, aber auch ein Metier, in dem Spezialisten aus der Übernahme solcher Risiken Profit zu schlagen versuchten. Engel geht es dabei nicht nur um die im engeren Sinne wirtschaftshistorische Dimension des Terminhandels, sondern auch um den gesellschaftlichen Umgang mit ökonomischen Risiken, um die Entwicklung ihrer wissenschaftlichen Analyse und ihrer publizistischen Kritik, schließlich um die damit zusammenhängende Regulierung des Börsenterminhandels und um die Terminbörse als Arbeitsplatz. In einem ausführlichen theoretischen Vorspann entwickelt er dazu anhand der unterschiedlichen Risikobegriffe Franklin H. Knights, Niklas Luhmanns und Ulrich Becks eine Typologie von Risikodimensionen, die eine Eingrenzung und zugleich historische Differenzierung der mit Termingeschäften verbundenen „Unsicherheitsstrukturen und Unsicherheitskulturen“ (S. 49) erlaubt.

Der Fokus der vier historischen Hauptkapitel und eines Ausblicks bis in die Gegenwart liegt sodann vor allem auf Deutschland und den USA, die als Beispiele zur Veranschaulichung bestimmter Trends zu verschiedenen Zeiten herangezogen werden. Das Buch leistet dadurch zugleich einen differenzierenden Beitrag zur Diskussion über die „Varieties of Capitalism“. Die Entstehung des modernen Terminhandels bis etwa 1870 beschreibt Engel als ,asymmetrische Raum-Zeit-Kompression“ (S. 97): Zwar beschleunigten Eisenbahn und Dampfschifffahrt auch die Güterströme des überregionalen und internationalen Handels, die Telegrafie erhöhte aber das Zirkulationstempo von Informationen noch viel stärker. Bei fungiblen, vor allem landwirtschaftlichen Handelsgütern (commodities), die sich durch Proben und Standards abbilden ließen, machte dies die Anwendung von Techniken des „Zeitkaufs“ sinnvoll, die zuvor im Wertpapier- und Effektenhandel entwickelt worden waren.

Ralf Ahrens $(\bowtie)$

Leibniz-Zentrum für Zeithistorische Forschung Potsdam, Potsdam, Deutschland

E-Mail: ahrens@zzf-potsdam.de 
Ihre Institutionalisierung an den Warenterminbörsen bewirkte eine Differenzierung und zugleich Vereinfachung des Umgangs mit Risiken.

$\mathrm{Zu}$ den vielen erhellenden Befunden des Buchs gehört, dass der Terminhandel zwar im Kontext der zunehmenden Globalisierung entstand, seine Entwicklung aber zeitweise quer zu der geläufigen Periodisierung lag: Der Erste Weltkrieg bremste seinen allgemeinen Aufschwung nur vorübergehend. Wie der internationale Vergleich zeigt, waren dafür im Einzelfall unterschiedliche Faktoren verantwortlich, die geografischen Schwerpunkte der Dynamik verlagerten sich ebenfalls mehrfach. Parallel dazu wuchs indes nicht nur die Kritik an den spekulativen, „spielerischen“ Verhaltensweisen mancher Marktteilnehmer, sondern auch die Neigung, durch Preisabsprachen und andere direkte Markteingriffe die Risiken, die der Terminhandel verringern sollte, vollständig auszuschalten. Substanzielle Regulierungs- und sogar Verbotsversuche gab es dabei scheinbar paradoxerweise gerade in den USA, die als Musterfall „liberaler“ Marktwirtschaften gelten. Vollkommen überflüssig wurden Warenterminbörsen schließlich in der nationalsozialistischen Preis- und Mengenbewirtschaftung, doch selbst an der Chicagoer Terminbörse ging das Geschäft unter verschärfter Regulierung und staatlicher Höchstpreisvorschriften massiv zurück.

In der Nachkriegszeit blieb der Terminhandel angesichts staatlicher Planungsansprüche und internationaler Stabilisierungsversuche zunächst ein „Nischenphänomen“ (S. 312). Sein Comeback begann in den 1970er Jahren, also in Zeiten der Desillusionierung über staatliche Steuerungskapazitäten und wachsender Volatilität auf vielen Märkten. Diese „Rückkehr des Risikos“ (S. 339) förderte zunächst die Entstehung neuer Warenterminmärkte in den USA (trotz zunehmender Regulierung). Die eigentliche Neuerung war jedoch der Siegeszug der financial futures durch die Ausdehnung des Terminkontrakthandels auf den Finanzsektor. Eine der zentralen Ursachen war das Ende des Währungsregimes von Bretton Woods, das die Risiken von Wechselkursänderungen drastisch erhöhte. Neben Währungskontrakten erlebte aber auch der neue Terminhandel mit Staats- und Unternehmensanleihen ebenso ein rasantes Wachstum wie das Geschäft mit Aktienoption. Engel zeigt hier überzeugend, welche Rolle der Aufstieg der Finanzmarktökonomie, aber auch die Populärkultur des tradings für eine „Konvergenz von Termin- und Wertpapiermärkten“ (S. 412) spielten, die von der realwirtschaftlichen Seite her keineswegs zwingend war.

Erst mit dem Aufstieg der daraus entstehenden Derivatebörsen - und parallel des außerbörslichen Derivatehandels - seit den 1990er Jahren sieht Engel dann tatsächlich jene „Risikoökonomie“ erreicht, von der der Buchtitel spricht: Das Termingeschäft dient nicht mehr primär der Einhegung von, sondern dem Handel mit Risiken, deren weltweite Kommodifizierung der Öffentlichkeit in der Finanzkrise 2007/08 besonders drastisch vor Augen geführt wurde. Diese Interpretation ist keineswegs deterministisch, sondern ganz im Gegenteil das Resultat einer konsequenten Historisierung von Marktprozessen und ihrer Beschreibung auf breiter Quellenbasis. Zu den Verdiensten des Buchs gehört es nicht zuletzt, die historische Kontingenz der Finanzialisierungsprozesse seit den 1970er Jahren (und damit auch ihre mögliche Umkehrbarkeit) herausgearbeitet zu haben.

Funding Open Access funding enabled and organized by Projekt DEAL. 
Open Access Dieser Artikel wird unter der Creative Commons Namensnennung 4.0 International Lizenz veröffentlicht, welche die Nutzung, Vervielfältigung, Bearbeitung, Verbreitung und Wiedergabe in jeglichem Medium und Format erlaubt, sofern Sie den/die ursprünglichen Autor(en) und die Quelle ordnungsgemäß nennen, einen Link zur Creative Commons Lizenz beifügen und angeben, ob Änderungen vorgenommen wurden.

Die in diesem Artikel enthaltenen Bilder und sonstiges Drittmaterial unterliegen ebenfalls der genannten Creative Commons Lizenz, sofern sich aus der Abbildungslegende nichts anderes ergibt. Sofern das betreffende Material nicht unter der genannten Creative Commons Lizenz steht und die betreffende Handlung nicht nach gesetzlichen Vorschriften erlaubt ist, ist für die oben aufgeführten Weiterverwendungen des Materials die Einwilligung des jeweiligen Rechteinhabers einzuholen.

Weitere Details zur Lizenz entnehmen Sie bitte der Lizenzinformation auf http://creativecommons.org/ licenses/by/4.0/deed.de. 